\title{
Energy Efficient Uplink Transmissions in LoRa Networks
}

\author{
Binbin Su, Zhijin Qin, Member, IEEE, Qiang Ni, Senior Member, IEEE
}

\begin{abstract}
LoRa has been recognized as one of the most promising low-power wide-area (LPWA) techniques. Since LoRa devices are usually powered by batteries, energy efficiency (EE) is an essential consideration. In this paper, we investigate the energy efficient resource allocation in LoRa networks to maximize the system EE (SEE) and the minimal EE (MEE) of LoRa users, respectively. Specifically, our objective is to maximize the corresponding $\mathrm{EE}$ by jointly exploiting user scheduling, spreading factor (SF) assignment, and transmit power allocations. To solve them efficiently, we first propose a suboptimal algorithm, including the low-complexity user scheduling scheme based on matching theory and the heuristic SF assignment approach for LoRa users scheduled on the same channel. Then, to deal with the power allocation, an optimal algorithm is proposed to maximize the SEE. To maximize the MEE of LoRa users assigned to the same channel, an iterative power allocation algorithm based on the generalized fractional programming and sequential convex programming is proposed. Numerical results show that the proposed user scheduling algorithm achieves near-optimal EE performance, and the proposed power allocation algorithms outperform the benchmarks.
\end{abstract}

Index Terms: energy efficiency, LoRa, low-power wide-area, matching theory.

\section{INTRODUCTION}

Driven by the massive connectivity, low data rate, and low power consumption requirements in the Internet of Things (IoT) networks, low-power wide-area (LPWA) networks have emerged as a potential solution to enable long-distance power efficient wireless communications [2], [3]. Compared with traditional technologies prevalent in IoT networks, such as Bluetooth, Wi-Fi, and Long-Term Evolution (LTE), LPWA techniques achieve better tradeoffs of coverage range, data rates, and power consumption. Among the emerging LPWA technologies, Long Range (LoRa) [4], which operates in the unlicensed bands, has attracted extensive attention.

LoRa network is composed of LoRa users, LoRa gateways, and the network server. It adopts typical star topology, in which the data and/or requests of LoRa end devices are collected by the LoRa gateway and then it forwards them to the LoRa network server [5]. The core of LoRa lies in the adopted chirp spread spectrum (CSS) technique and multiple

Part of this work [1] was presented at the IEEE Global Communications Conference (GLOBECOM 2018), Abu Dhabi, UAE, Dec. 2018.

Binbin Su and Qiang Ni are with the School of Computing and Communications, Lancaster University, Lancaster LA1 4WA, U.K. (emails: b.su@lancaster.ac.uk; q.ni@lancaster.ac.uk)

Zhijin Qin is with the School of Electronic Engineering and Computer Science, Queen Mary University of London, London E1 4NS, U.K. (email: z.qin@qmul.ac.uk) orthogonal spreading factors (SFs). The system throughput is enhanced as multiple LoRa end devices can transmit at the same time and frequency slot in one channel, but with different SFs. Different SFs result in diverse signal to noise ratio (SNR) sensitives, which leads to different transmission rates and coverage ranges. So far, extensive research has been carried out to investigate the impact of perfect and imperfect SF orthogonality. For instance, the authors in [6] have adopted the stochastic geometry tool to analyze the cochannel interference caused by LoRa users using the same $\mathrm{SF}$ over the same channel. Besides, the influence of imperfect SF orthogonality on the system throughput has been analyzed in [7] to provide insights on the SF assignment design of uplink LoRa networks. The packet loss caused by inter-SF collisions has been numerically analyzed and then validated with experiments based on commercial devices in [8]. The joint SF assignment and transmit power allocation algorithm has been investigated in [9] to improve throughput fairness by considering both co-SF and inter-SF interferences.

Typically, adaptive data rate (ADR) mechanism can be enabled in the LoRaWAN to adjust the SF assignment and transmit power, based on the messages obtained from previous uplink measurements [10]. The ADR mechanism has been presented in [11] from the perspective of the average coverage time. The near-far problem and fair data rate deployment ratios have been addressed in [12] to achieve the data rate fairness among LoRa nodes based on LoRaWAN. By taking the uplink throughput and data transmission times of a single end device as the performance metrics, the authors in [13] have analyzed the capacity and scalability performance for uplink LoRaWAN. However, the centralized ADR scheme is inefficient as it requests a number of uplink and downlink information exchanges to update the transmit power and SF step by step. Moreover, ADR is unable to deal with user collisions, which boosts the need to design efficient user scheduling and power allocation schemes. Besides, the performance of a LoRa network has been investigated in [14] to guarantee the fairness among LoRa users, with particular focus on the effects of interference caused by LoRa users using the same SF. By adopting an interference-based simulation model, the authors in [15] have analyzed the LoRa scalability, i.e., the number of end devices that can be served per LoRa gateway. It is worth noting that all the above studies mainly focus on the system capacity, scalability, and interference management for uplink LoRa networks.

Moreover, energy efficiency (EE) is considered as an essential measurement metric in communication system design from the perspective of both operators and users [16]-[20]. 
Thus far, extensive works have been conducted to realize energy efficient communications for various networks. For example, the joint time allocation and power control scheme has been investigated in [21] to improve the $\mathrm{EE}$ of the wireless powered communication networks. The energy efficient beamforming design has been studied in [22] to maximize the EE for multiple-input single-output (MISO) NOMA systems. The polynomial-time algorithm has been proposed in [23] to tackle the EE maximization problem for orthogonal frequencydivision multiple access (OFDMA) networks with base station coordination. To guarantee fairness among users, the joint subcarrier assignment and power allocations have been exploited in [24] to maximize the worst-link EE of OFDMA systems. Particularly, energy consumption becomes a key point in IoT networks [25], since most IoT applications are deployed on battery-powered end devices that require a long lifetime, i.e., over 10 years. In [26], a novel deployment scheme based on clustering topology has been proposed to realize the energy efficient IoT networks. However, different from the above networks, the energy efficient design in LoRa involves not only user scheduling and power allocation, but also SF assignment. Therefore, the existing EE methods cannot be straightforwardly applied in LoRa networks.

Furthermore, LoRa networks feature low power operation to enable long lifetime and high maintenance costs of massive nodes, hence EE is more critical to be considered compared with cellular networks. In practice, different communication system requirements result in diverse EE design models. On the one hand, to reduce the energy consumption of the entire system, it is desired to maximize the system EE (SEE) [21], [23], [27]. SEE design allocates more resources to users with better channel conditions, hence the performance improvement is achieved at the cost of LoRa users experiencing worse channel conditions, which arises unfairness issues among LoRa users. To guarantee the EE fairness, the problem can be formulated into the format of max-min EE (MEE) [24]. Both EE models provide imperative and normative references for energy efficient LoRa design.

To the best of our knowledge, the EE aspect of resource allocation has not been well studied for LoRa networks, from the perspective of SEE and MEE. Motivated by the above observations and the fact that uplink communication is highly favored, we investigate energy efficient transmission design for uplink LoRa networks in this paper.

The main contributions of this paper are summarized as follows:

1) To thoroughly emphasize the EE issues in the uplink transmission of LoRa networks, we formulate the optimization problems in terms of SEE and MEE, respectively. Then we decompose the original nonconvex problems into three sub-problems correspondingly by investigating the joint optimization problem of user scheduling, SF assignment, and power allocations.

2) For the formulated problems, we first propose a distributed scheme by formulating user scheduling as a two-sided matching problem, which facilitates LoRa users to realize self-matching with proper channels. For LoRa users matched into the same channel, we propose a low-complexity heuristic SF assignment algorithm to provide better coverage.

3) Within each channel, the LoRa gateway performs centralized algorithms to optimize power for LoRa users. Specifically, to maximize SEE, the objective is first approximated by its lower bound, then an optimal transmit power allocation algorithm is further proposed. For the case of MEE, we first transform it into a more tractable form based on the generalized fractional programming method. Then the iterative power allocation problem is reformulated as the difference of convex (DC) functions, which is efficiently solved via sequential convex programming.

The remainder of this paper is organized as follows. In Section II, we present the system model of the uplink LoRa networks and formulate two EE optimization problems. The low-complexity energy efficient user scheduling and heuristic SF assignment schemes are developed in Section III. In Section IV, the power allocation algorithms for both problems are provided. Simulation results are presented in Section V and finally the paper is concluded in Section VI.

\section{System Model AND Problem Formulation}

\section{A. System Model}

Considering the uplink transmission in LoRa networks, $N$ active LoRa users communicate with one LoRa gateway through $M$ channels, which is shown in Fig. 1. LoRa users located within the same channel share the same time and frequency slots by adopting different SFs. Both the LoRa gateway and LoRa users are equipped with the single antenna. Denote $\boldsymbol{M}=\{1, \ldots M\}$ and $\boldsymbol{N}=\{1, \ldots N\}$ to be the channels set and users set, respectively. $B_{m} \mathrm{~Hz}$ is the bandwidth of the $m$-th channel, $S C_{m}$. The number of LoRa users scheduled on $S C_{m}$ is labelled as $S_{m}$, i.e., $S_{m}=\sum_{l=1}^{N} s_{m, l}$, where $s_{m, l} \in\{0,1\}$ is used to indicate whether an arbitrary user, $U_{l}$, is allocated to $S C_{m}$. If $s_{m, l}=1$, it indicates that $U_{l}$ occupies $S C_{m}$, and $s_{m, l}=0$ if otherwise. Let $\boldsymbol{S}=\left\{s_{m, l} \mid m \in \boldsymbol{M}, l \in\right.$ $N\}$ denote the set of user clustering. It is worth mentioning that $S_{m}$ should be no more than 6 as the available SFs range from 7 to 12 [28], which limits the maximum number of active LoRa users that can be served simultaneously in one channel. Let $p_{m, l}$ be the power allocated to $U_{l}$ using $S C_{m}$ and $\boldsymbol{P}=\left\{p_{m, l} \mid m \in \boldsymbol{M}, l \in \boldsymbol{N}\right\}$ represent the set of power allocation coefficients. It is assumed that perfect channel state information (CSI) is known at the LoRa gateway by containing the CSI feedbacks in the uplink message.

Assuming that $S_{m}$ users are allocated within $S C_{m}$, the signal received at the LoRa gateway through $S C_{m}$ can be expressed as

$$
y_{m}=\sum_{l=1}^{S_{m}} s_{m, l} \sqrt{p_{m, l}} h_{m, l}+z_{m},
$$

where $h_{m, l}=g_{m, l} \varsigma_{m} d_{l}^{-a}$ indicates the channel coefficient between $U_{l}$ and the LoRa gateway occupying $S C_{m}, g_{m, l}$ represents the Rayleigh fading channel gain, $d_{l}$ denotes the distance from $U_{l}$ to the LoRa gateway, $a$ is the channel path 


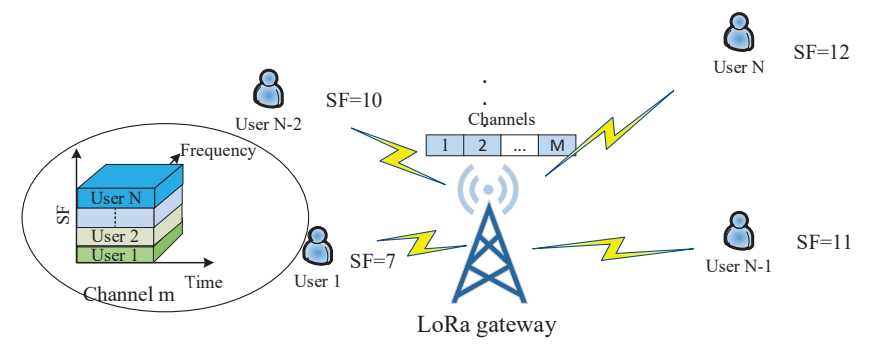

Fig. 1. System model of resource allocation in LoRa networks.

loss exponent, and $\varsigma_{m}$ is a constant depending on path loss of $S C_{m}$. Denote $z_{m} \in \boldsymbol{C N}\left(0, \sigma_{m}^{2}\right)$ as the additive white Gaussian noise (AWGN) with noise variance $\sigma_{m}^{2}$.

The signal-to-interference-plus-noise ratio (SINR) of $U_{l}$, received at the gateway over $S C_{m}$ is characterized as

$$
\operatorname{SINR}_{m, l}(\boldsymbol{S}, \boldsymbol{P})=\frac{s_{m, l} p_{m, l}\left|h_{m, l}\right|^{2}}{I_{S F}+\sigma_{m}^{2}}
$$

where $I_{S F}=\sum_{k=1, k \neq l}^{S_{m}} p_{m, k}\left|h_{m, k}\right|^{2} \psi(l, k)$ represents the interference caused by LoRa users adopting different SFs in the same channel, and $\psi \in[0,1]$ represents the cross correlation factors between the coded LoRa waveforms with different SFs. Note that the interference is introduced due to imperfect SF orthogonality.

Similar to [29], we assume that a transmission rate of Shannon's upper bound can be achieved by perfect coding, and Shannon rate is adopted to model the LoRa-specific rates for mathematical tractability. Note that due to the limited coding rates in LoRa, the adopted Shannon rate serves as an upper bound for the energy efficient LoRa design. The achievable data rate and the overall power consumption for the $l$-th LoRa user over $S C_{m}$ can be denoted as follows

$$
\left\{\begin{array}{l}
R_{m, l}(\boldsymbol{S}, \boldsymbol{P})=B_{m} \log _{2}\left(1+\mathrm{SINR}_{m, l}\right), \\
P_{m, l}(\boldsymbol{S}, \boldsymbol{P})=\zeta_{m, l} p_{m, l}+P_{c}^{l}
\end{array}\right.
$$

where $\zeta_{m, l} \geq 1$ is a constant denoting the power inefficiency, $P_{c}^{l}$ represents the additional circuit power consumption of $U_{l}$ owing to inevitable electronic operations [23].

Therefore, the achievable sum rate and the total power consumption of the system can be expressed as

$$
\left\{\begin{array}{l}
R(\boldsymbol{S}, \boldsymbol{P})=\sum_{m=1}^{M} \sum_{l=1}^{S_{m}} R_{m, l}(\boldsymbol{S}, \boldsymbol{P}), \\
P(\boldsymbol{S}, \boldsymbol{P})=\sum_{m=1}^{M} \sum_{l=1}^{S_{m}} P_{m, l}(\boldsymbol{S}, \boldsymbol{P}) .
\end{array}\right.
$$

For energy efficient uplink transmissions in LoRa networks, the goal is to maximize SEE, which is defined as information bits within a unit energy. Therefore, SEE is formulated as a ratio of the system sum rate to the total power consumption, which can be characterized as

$$
\eta=\frac{R(\boldsymbol{S}, \boldsymbol{P})}{P(\boldsymbol{S}, \boldsymbol{P})} \text {. }
$$

Moreover, MEE is defined as the ratio of individual LoRa user rate to the corresponding consumed power, which can be given as

$$
\eta_{m, l}=\frac{R_{m, l}(\boldsymbol{S}, \boldsymbol{P})}{P_{m, l}(\boldsymbol{S}, \boldsymbol{P})}
$$

\section{B. Problem Formulations}

To acquire an energy efficient resource allocation design for the considered LoRa networks, we formulate the EE optimization problems based on two major performance measurement criteria in terms of SEE and MEE, respectively.

1) SEE: The SEE maximization problem is formulated as follows

$$
\begin{aligned}
& \text { (P1) } \max _{\boldsymbol{S}, \boldsymbol{P}, \tau_{S F}} \eta \text {, } \\
& \text { s.t. } \quad 0 \leq p_{m, l} \leq p_{\max } \text {, } \\
& s_{m, l} \in\{0,1\}, \forall m, l, \\
& \sum_{m=1}^{M} s_{m, l} \leq 1, \forall l \text {, } \\
& \sum_{l=1}^{N} s_{m, l} \leq \Lambda_{\max }, \forall m, \\
& \frac{p_{m, l}\left|h_{m, l}\right|^{2}}{\sigma_{m}^{2}} \geq \tau_{S F}, \forall m, l .
\end{aligned}
$$

In (P1), (7a) represents the formulated problem to maximize SEE. Constraint (7b) limits the transmit power of each LoRa user. In (7c), the value of the user cluster indicator $s_{m, l}$ is either 0 or 1 . Constraint (7d) indicates that each LoRa user can access at most one channel. Due to the maximum number of available SF and interference control, it is assumed that at most $\Lambda_{\max }$ users can be assigned to the same channel, which is guaranteed by (7e). It is worth noting that the desired LoRa user adopting a given SF can be detected successfully only if the received SNR is no less than the threshold $\tau_{S F}$, which is guaranteed by constraint (7f). Table I [6] shows the relationship between the required $\mathrm{SNR}$ and $\mathrm{SF}$.

2) $M E E$ : The MEE optimization problem is given by

$$
\begin{gathered}
\left(\mathbf{P 1}{ }^{\prime}\right) \underset{\boldsymbol{S}, \boldsymbol{P}, \tau_{S F}}{\max } \min _{m, l} \eta_{m, l}, \\
\text { s.t. } \quad(7 \mathrm{~b})-(7 \mathrm{f}),
\end{gathered}
$$

where (8a) denotes the objective to maximize MEE.

Theorem 1. The formulated problems of both $\boldsymbol{P 1}$ and $\boldsymbol{P 1}$ are NP-hard.

Proof: The proof is provided in Appendix A.

Since the formulated problems are nonconvex and NP-hard, it is challenging and intractable to solve $(\mathbf{P 1})$ and $\left(\mathbf{P 1} \mathbf{\prime}^{\prime}\right)$ within polynomial time.

Furthermore, the current ADR mechanism adopted in LoRaWAN fails to perform the channel selection effectively, a more efficient distributed user scheduling scheme needs to be designed. Besides, the ADR scheme optimizes the transmit power and SF based on some previous uplink messages, which achieves low resource efficiency. Therefore, the optimal transmit power allocation scheme that can be easily implemented at the LoRa gateway is required. Moreover, as can be observed from the expression of the objective functions and constraint (7f), the channel and power allocations are coupled with each other for both MEE and SEE. As the formulated problem is NP-hard, to avoid the considerable complexity of the global 
TABLE I: Relationship between distance range and spreading factors [14].

\begin{tabular}{|l|l|l|l|l|l|l|}
\hline Spreading factor (SF) & 7 & 8 & 9 & 10 & 11 & 12 \\
\hline Distance Range $(\mathrm{km})$ & 2 & 4 & 6 & 8 & 10 & 12 \\
\hline Required SNR $(\mathrm{dB})$ & -7.5 & -10 & -12.5 & -15 & -17.5 & -20 \\
\hline
\end{tabular}

optimum solution, we will exploit user scheduling, SF assignment, and power allocation schemes separately. Specifically, LoRa users first perform self-matching to match with the corresponding channels. Since SF is only constrained by the linear inequality (7f), for a user satisfying the SNR threshold with a given SF may use any higher SF, as shown in Table I. In this case, if there are multiple users satisfying the same SNR threshold at the same time, the optimal channel, power and SF allocation can only be achieved by the exhaustive search method. To reduce the complexity, a suboptimal SF assignment algorithm is proposed to be operated at the LoRa gateway. Finally, the LoRa gateway allocates power for LoRa users sharing the same channel, on the basis of the proposed optimal power allocation algorithms. The details are described in the next two sections.

\section{ENERGY-EFFICIENT USER SCHEDULING}

In this section, for the two formulated problems, energy efficient user scheduling scheme is firstly proposed to maximize the corresponding EE. For LoRa users scheduled on the same channel, a heuristic distance-based SF assignment scheme is then proposed.

\section{A. User Scheduling}

In this section, we provide a matching theory based user scheduling scheme with low complexity. Firstly, by assuming that each user is allocated with the maximum power and a given SF, (P1) and $\left(\mathbf{P 1}{ }^{\prime}\right)$ can be reformulated as

$$
\begin{aligned}
& \text { (P2) } \max _{S} \eta \text {, } \\
& \text { or } \max _{\boldsymbol{S}} \min _{m, l} \eta_{m, l} \text {, } \\
& \text { s.t. } s_{m, l} \in\{0,1\} \text {, } \\
& \sum_{m=1}^{M} s_{m, l} \leq 1, \forall l \text {, } \\
& \sum_{l=1}^{N} s_{m, l} \leq \Lambda_{\max }, \forall m .
\end{aligned}
$$

It is noted that (P2) is a many-to-one matching problem for both SEE and MEE, as at most one channel can be allocated to a LoRa user while a subset of LoRa users can be assigned into the same channel. Moreover, due to the interference term in (2), each user's preference on the channel is not only influenced by the channel conditions, but also impacted by the other LoRa users sharing the same channel. Similarly, each channel not only cares for which LoRa users to match with, but also concerns the co-channel interference introduced by the other subset of LoRa users with different SFs. Hence, this is a many-to-one matching game with peer effects [30].

To better illustrate the matching model with peer effects, we firstly introduce a preference ordering for LoRa users, in which for any given user $U_{l} \in \boldsymbol{N}$, any two channels $S C_{m}$, $S C_{m^{\prime}} \in \boldsymbol{M}$, any two matchings $\varphi$ and $\varphi^{\prime}$ are defined as

$$
\left(S C_{m}, \varphi\right) \succ_{U_{l}}\left(S C_{m^{\prime}}, \varphi^{\prime}\right) \Leftrightarrow R_{m, l}(\varphi)>R_{m, l}\left(\varphi^{\prime}\right),
$$

which means that LoRa user $U_{l}$ prefers channel $S C_{m}$ in $\varphi$ rather than $S C_{m^{\prime}}$ in $\varphi^{\prime}$ only if $U_{l}$ achieves higher rate over channel $S C_{m}$ than over $S C_{m^{\prime}}$. It is worth mentioning that the preference order is based on achievable rates. The reason is that, with given transmit power allocation, the power consumption in the denominator of the objective is a fixed value, the EE objectives are equivalent to the corresponding rates optimization problems.

By defining channel $S C_{m}$ 's preference value on the user set as $R_{m}^{S E E}=\sum_{l=1}^{N} R_{m, l}$ and $R_{m}^{M E E}=\min R_{m, l}, \forall l \in S C_{m}$ for SEE and MEE, respectively, the preference ordering for channel $S C_{m}$ can be obtained similarly.

Due to the existence of peer effects, stable matching is not straightforwardly guaranteed. Therefore, the two-sided exchange stability has been introduced to depict the impact of peer effects on the matching game [30]. Firstly, a swap matching and swap blocking pair are defined as follows:

Definition 1. A swap matching behaviour $\varphi_{l}^{j}=$ $\varphi \backslash\left\{\left(U_{l}, S C_{m}\right),\left(U_{j}, S C_{n}\right)\right\} \quad \cup \quad\left\{\left(U_{j}, S C_{m}\right),\left(U_{l}, S C_{n}\right)\right\}$ is defined as $\varphi\left(U_{l}\right)=S C_{m}$ and $\varphi\left(U_{j}\right)=S C_{n}$.

Note that a swap matching is realized by performing a swap operation, which motivates us to introduce swap-blocking pair.

Definition 2. Given a matching $\varphi$ with a pair $\left(U_{l}, U_{j}\right)$, if there exists $\varphi\left(U_{l}\right)=S C_{m}$ and $\varphi\left(U_{j}\right)=S C_{n}$ such that

1) $\forall q \in\left\{U_{l}, U_{j}, S C_{m}, S C_{n}\right\}, \varphi_{l}^{j}(q) \geq_{q} \varphi(q)$;

2) $\exists q \in\left\{U_{l}, U_{j}, S C_{m}, S C_{n}\right\}, \varphi_{l}^{j}(q) \succ_{q} \varphi(q)$,

which means the swap matching $\varphi_{l}^{j}$ is approved, and we call $\left(U_{l}, U_{j}\right)$ as a swap-blocking pair in $\varphi$.

The definition demonstrates that the achievable data rates of any player, i.e., $U_{l}$ and $S C_{m}$, will not decrease by employing a swap matching, and the data rates of at least one player will increase. Then a stable matching status can be achieved through a set of swap matching operations, known as a twosided exchange stable matching that is described in Definition 3.

Definition 3. A two-sided exchange stable (2ES) matching $\varphi$ can be achieved if it is not blocked by any swap-blocking pair.

Based on the above definition, the proposed user scheduling algorithm is described as Algorithm 1, which consists of initialization step and swap matching step. The initialization step is a deferred acceptance algorithm [31], which aims to generate the initial matching. Specifically, the CSI-based preference list is constructed for each LoRa user, i.e., $j^{*}=\underset{j \in \boldsymbol{M}}{\operatorname{argmax}}\left|h_{j, i}\right|^{2}$ is the most preferred channel for LoRa user $U_{i}$. For the LoRa gateway, it constructs the distance-based preference list, i.e., the highest preference is the closest LoRa user, due to the fact that LoRa provides long-range communications. Each LoRa user proposes to the highest preference channel, and each channel picks at most $\Lambda_{\max }$ users based on its preference list. Then the 
remaining LoRa users propose to their second preference, and the process stops until no unmatched users exist. In the swap matching step, each LoRa user keeps searching for swap-blocking pairs to perform swap matching operation if approved. The searching terminates until no swap-blocking pairs can be formed, and the final stable matching is returned.

Theorem 2. The proposed user scheduling Algorithm 1 converges to a $2 E S$ matching $\varphi^{*}$ within a finite number of swap operations.

Proof: The proof is provided in Appendix B.

Theorem 3. The computational complexity of Algorithm 1 is $O\left(M N+\frac{1}{2} I \Lambda_{\max } N(M-1)\right)$ at worst, where I denotes the number of iterations for swap matching step.

Proof: The proof is provided in Appendix C.

\section{B. SF Assignment}

As can be seen from the structure of the formulated problems, i.e., $(\mathbf{P 1})$ and $\left.(\mathbf{P 1})^{\prime}\right)$, the SF is only related to the linear inequality constraint, i.e., the SNR threshold requirement. With given power allocation and user scheduling scheme, we need to check whether the SF constraint is satisfied. By applying the proposed user scheduling scheme, LoRa users are allocated into the corresponding channels. For a given channel, the distance of each scheduled LoRa user is easily obtained at the LoRa gateway. Since LoRa network aims to realize long-range communications up to $40 \mathrm{~km}$, the largescale fading becomes the main effect for the channel gain. Therefore, a heuristic distance-based SF assignment scheme is proposed, which is summarized as Algorithm 2. Specifically, in each channel, a predefined SF is assigned to each LoRa user according to the relationship between the distance range and corresponding SF based on Table I. Then the LoRa gateway keeps searching for the SF assigned to more than one LoRa user. For LoRa users sharing the same SF, a higher available $\mathrm{SF}$ in the network is reassigned to the one with a longer distance to the LoRa gateway. The searching iteration stops until all LoRa users accessing the same channel occupy a unique SF.

\section{ENERGY-EFFICIENT POWER ALLOCATION ALGORITHMS}

In this section, we focus on the optimal power allocations to maximize SEE and MEE, respectively, with the obtained user scheduling and SF assignment schemes given in the last section. It is worth mentioning that though the proposed power allocation algorithms are centralized approaches, it is easy to be implemented at the LoRa gateway. The reason is that, the LoRa gateway can easily obtain the CSI of LoRa users assigned within each channel, due to the fact that the number of LoRa users sharing the same channel is limited after user scheduling. Moreover, as the LoRa gateway is more powerful than LoRa users, the centralized power allocation algorithms implemented at the LoRa gateway help LoRa users avoid energy consumption for power allocation. $\overline{\text { Algorithm } 1 \text { User Scheduling Algorithm for LoRa Networks }}$ Based on Matching Theory

\section{Initialization step}

While there exists unmatched users and channels 1) $j^{*}=\underset{j \in M}{\operatorname{argmax}}\left|h_{j, i}\right|^{2}$.

2) LoRa user $U_{i}$ matches with its most preferred channels that it has not been rejected before.

3) Remove $U_{i}$ from $N$.

Swap matching step

Repeat

1) For user $U_{i} \in N$, it searches $N \backslash U_{j}$.

2) if $U_{i}$ and $U_{j}$ is swap-blocking pair, then $U_{i}$ exchanges its matching with $U_{j}$ and set $\varphi=\varphi_{i}^{j}$.

3) else

4) keep the current matching.

5) end if

Until no swap-blocking pair can be formed for all users. Return the stable matching $\varphi$

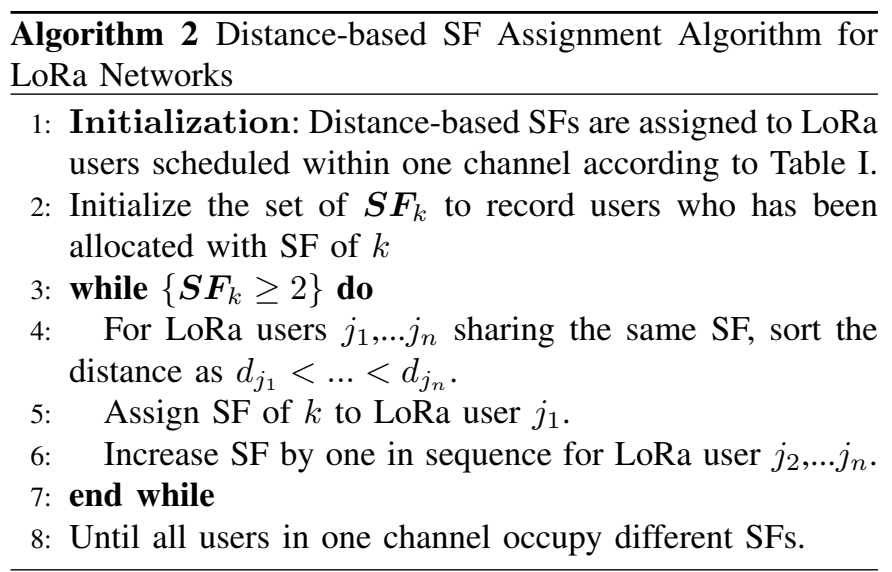

\section{A. Energy-Efficient Power Allocation for SEE}

With given user scheduling and SF assignment schemes, we consider the power allocation problem (P1) with constraints (7b) and (7f). The difficulty lies in the nonconvex objective function as all the constraints are linear inequalities. We first approximate the objective with the lower bound by using the following inequality [32]

$$
\ln (1+\gamma) \geq \alpha \ln \gamma+\beta
$$

where

$$
\begin{gathered}
\alpha=\frac{\tilde{\gamma}}{1+\tilde{\gamma}}, \\
\beta=\ln (1+\tilde{\gamma})-\frac{\tilde{\gamma}}{1+\tilde{\gamma}} \ln (\tilde{\gamma}) .
\end{gathered}
$$

The approximation is tight when $\gamma=\tilde{\gamma}$. The proof can be easily acquired by substituting $\gamma=\tilde{\gamma}$ into the right side of inequality (11). 
With inequality (11), we get a lower bound for the achievable data rate of $U_{l}$ accessing $S C_{m}$ as follows

$$
\begin{aligned}
& R_{m, l} \geq \tilde{R}_{m, l}=\frac{B_{m}}{\ln 2}\left(\alpha_{m, l} \ln \left(\operatorname{SINR}_{m, l}\right)+\beta_{m, l}\right) \\
& =\frac{B_{m}}{\ln 2}\left(\alpha_{m, l} \ln \left(\frac{p_{m, l}\left|h_{m, l}\right|^{2}}{\sum_{k=1, k \neq l}^{S_{m}} p_{m, k}\left|h_{m, k}\right|^{2}+\sigma_{m}^{2}}\right)+\beta_{m, l}\right) .
\end{aligned}
$$

However, $\tilde{R}_{m, l}$ is still nonconvex. To convert it into a concave expression, we introduce a variable transformation as $x_{m, l}=\ln \left(p_{m, l}\right)$. Then we have

$$
\begin{array}{r}
\ln \left(\operatorname{SINR}_{m, l}\right)=\ln \left(\left|h_{m, l}\right|^{2}\right)+x_{m, l} \\
-\ln \left(\sum_{k=1, k \neq l}^{S_{m}} e^{x_{m, k}}\left|h_{m, k}\right|^{2}+\sigma_{m}^{2}\right),
\end{array}
$$

which is concave over $\boldsymbol{x}=\left\{x_{m, l} \mid m \in \boldsymbol{M}, l \in\right.$ $N\}$ since the log-sum-exp function is convex. Through the above transformation, the original objective can then be approximated by its lower bound function, i.e., $\eta \geq \tilde{\eta}=\frac{\sum_{m=1}^{M} \sum_{l=1}^{S_{m}} \frac{B_{m}}{\ln 2}\left(\alpha_{m, l} \ln \left(\operatorname{SINR}_{m, l}\right)+\beta_{m, l}\right)}{\sum_{m=1}^{M} \sum_{l=1}^{S_{m}}\left(\zeta_{m, l} p_{m, l}+P_{c}^{l}\right)}$. The lower bound approximation is a concave-convex fractional function as it consists of a concave numerator $f\left(p_{m, l}\right)=$ $\sum_{m=1}^{M} \sum_{l=1}^{S_{m}} \frac{B_{m}}{\ln 2}\left(\alpha_{m, l} \ln \left(\operatorname{SINR}_{m, l}\right)+\beta_{m, l}\right)$, and an affine denominator $g\left(p_{m, l}\right)=\sum_{m=1}^{M} \sum_{l=1}^{S_{m}}\left(\zeta_{m, l} p_{m, l}+P_{c}^{l}\right)$. The concave-convex fractional problem can be efficiently solved with the CharnesCooper transformation [33], which is given as follows:

Lemma 1. A concave-convex fractional problem, max $\frac{f(x)}{g(x)}$, where $f$ is concave and $g$ is convex, can be reformulated as a concave problem

$$
\begin{aligned}
& \max \quad \phi f\left(\frac{y}{\phi}\right) \\
& \text { s.t. } \quad \phi g\left(\frac{y}{\phi}\right) \leq 1,
\end{aligned}
$$

with the Charnes-Cooper transformation $y=\frac{x}{g(x)}, \phi=\frac{1}{g(x)}$ and $\phi>0$.

As a result, the original problem is recast as the following equivalent convex optimization problem

$$
\begin{aligned}
\text { (P3) } \max _{\tilde{x}_{m, l}} \quad & \phi \sum_{m=1}^{M} \sum_{l=1}^{S_{m}} \tilde{R}_{m, l}\left(\frac{\tilde{x}_{m, l}}{\phi}\right) \\
\text { s.t. } \quad & \phi\left(\sum_{m=1}^{M} \sum_{l=1}^{S_{m}} e^{\frac{\tilde{x}_{m, l}}{\phi}}+P_{c}\right) \leq 1 \\
& \phi e^{\frac{\tilde{x}_{m, l}}{\phi}} \geq 0 \\
& \phi\left(e^{\frac{\tilde{x}_{m, l}}{\phi}}-p_{\max }\right) \leq 0, \forall m, l \\
& \phi\left(\sigma_{m}^{2} \tau_{S F}-e^{\frac{\tilde{x}_{m, l}}{\phi}}\left|h_{m, l}\right|^{2}\right) \leq 0, \forall m, l, \\
& \phi>0
\end{aligned}
$$

\section{Algorithm 3 Optimal Power Allocation for Solving (P3)}

Initialize feasible power allocation variables $\boldsymbol{P}^{\mathbf{1}}$.

Set $t=1$. The value of (16a) is calculated with $\boldsymbol{P}^{\mathbf{1}}$, denoted as $\eta^{0}$.

while $\frac{\eta^{t}-\eta^{t-1}}{\eta^{t-1}}>\epsilon$, where $\epsilon$ is a given constant.

Set $t \stackrel{\eta}{=} t+1$.

Update $\tilde{x}_{m, l}^{t}$ and $\phi^{t}$ by solving (P3).

Update power allocation variables $\boldsymbol{P}^{t}$ by $p_{m, l}^{t}=e^{\frac{\tilde{x}_{m, l}^{t}}{\phi^{t}}}$.

Update the objective value $\eta^{t}$.

\section{end while}

Output the optimal $\boldsymbol{P}^{*}$.

where

$$
\begin{aligned}
& \tilde{R}_{m, l}\left(\frac{\tilde{x}_{m, l}}{\phi}\right)=\frac{B_{m}}{\ln 2}\left(\alpha_{m, l} \ln \left(\operatorname{SINR}_{m, l}\right)+\beta_{m, l}\right), \\
& \ln \left(\operatorname{SINR}_{m, l}\right)=\ln \left(\frac{e^{\frac{\tilde{x}_{m, l}}{\phi}}\left|h_{m, l}\right|^{2}}{\sum_{k=1, k \neq l}^{S_{m}} e^{\frac{\tilde{x}_{m, k}}{\phi}}\left|h_{m, k}\right|^{2}+\sigma_{m}^{2}}\right) .
\end{aligned}
$$

The equivalence between (14) and (17b) is guaranteed with the aid of Charnes-Cooper transformation introduced by Lemma 1. It is noted that $(\mathbf{P 3})$ is a convex problem, which can be efficiently solved with standard convex solvers [34]. The corresponding procedure is outlined in Algorithm 3.

To prove the convergence of the proposed Algorithm 3, we provide the following proposition.

Proposition 1. The value of $\eta$ is improved continuously in each iteration of Algorithm 3, and finally Algorithm 3 converges to a Karush-Kuhn-Tucker (KKT) point of the original problem.

\section{Proof: The proof is provided in Appendix D.}

\section{B. Energy-Efficient Power Allocation for MEE}

For the case of MEE, given user scheduling and SF assignment, denote $\eta_{M E E}^{o p t}$ and $\boldsymbol{P}^{o p t}$ as the optimal solution to MEE and power allocation coefficients, the following results hold

$$
\eta_{M E E}^{o p t}=\max _{\boldsymbol{P}} \min _{m, l} \frac{R_{m, l}(\boldsymbol{P})}{P_{m, l}(\boldsymbol{P})}=\min _{m, l} \frac{R_{m, l}\left(\boldsymbol{P}^{o p t}\right)}{P_{m, l}\left(\boldsymbol{P}^{o p t}\right)} .
$$

Then we have Theorem 4 as below:

Theorem 4. The optimal solution $\eta_{M E E}^{o p t}$ is obtained on condition that

$$
\begin{aligned}
& \max _{\boldsymbol{P}} \min _{m, l}\left[R_{m, l}(\boldsymbol{P})-\eta_{M E E}^{o p t} P_{m, l}(\boldsymbol{P})\right] \\
= & \min _{m, l}\left[R_{m, l}\left(\boldsymbol{P}^{o p t}\right)-\eta_{M E E}^{o p t} P_{m, l}\left(\boldsymbol{P}^{o p t}\right)\right]=0 .
\end{aligned}
$$

Proof: The proof is provided in Appendix E.

Theorem 4 indicates that the optimal solutions of the original problem can be obtained by equivalently solving (19). As the value of $\eta_{M E E}^{o p t}$ is unknown in advance, the properties of (19) need to be further revealed. 
Denote that $\pi\left(\eta_{m, l}\right)=\max _{\boldsymbol{P}} \min _{m, l}\left[R_{m, l}(\boldsymbol{P})-\eta_{m, l} P_{m, l}(\boldsymbol{P})\right]$, we have the following theorem:

Theorem 5. i) $\pi\left(\eta_{m, l}\right)$ strictly decreases with $\eta_{m, l}$.

ii) With $\eta_{m, l}>0$, we obtain that

$$
\pi\left(\eta_{m, l}\right)=\left\{\begin{array}{lll}
>0 & \text { if } & \eta_{m, l}<\eta_{M E E}^{o p t}, \\
=0 & \text { if } & \eta_{m, l}=\eta_{M E E}^{o p t}, \\
<0 & \text { if } & \eta_{m, l}>\eta_{M E E}^{o p t}
\end{array}\right.
$$

Proof: The proof is provided in Appendix F.

Therefore, based on the properties of $\pi\left(\eta_{m, l}\right)$, we can apply the bisection method to solve it. The initial lower and upper bounds can be set as $\eta_{m, l}^{\min }=0$ and $\eta_{m, l}^{\max }$, where $\eta_{m, l}^{\max }$ is a relatively large constant. Given $\eta_{m, l}^{\min }$ and $\eta_{m, l}^{\max }$, the algorithm based on the bisection method is summarized as Algorithm 4.

For the $i$-th iteration with a given $\eta_{m, l}^{i}$ at line 4 of Algorithm 4 , to update the power allocation coefficients, we need to deal with the following optimization problem:

$$
\begin{aligned}
\text { (P4) } \max _{\boldsymbol{P}} \min _{m, l} R_{m, l}(\boldsymbol{P})-\eta_{m, l}^{i} P_{m, l}(\boldsymbol{P}), \\
\text { s.t. } \quad 0 \leq p_{m, l} \leq p_{\max }, \\
\frac{p_{m, l}\left|h_{m, l}\right|^{2}}{\sigma_{m}^{2}} \geq \tau_{S F}, \forall m, l .
\end{aligned}
$$

All the constraints in (P4) are convex, the difficulty lies in the nonconvex objective. To deal with it, an auxiliary variable $\mu$ is introduced to denote that $\min _{m, l} R_{m, l}(\boldsymbol{P})-$ $\eta_{m, l}^{i} P_{m, l}(\boldsymbol{P}) \geq \mu$. Due to the minimization operator, $R_{m, l}(\boldsymbol{P})-\eta_{m, l}^{i} P_{m, l}(\boldsymbol{P}) \geq \mu$ can be satisfied for all LoRa users. For a given $\eta_{m, l}^{i}$, the problem is reformulated as

$$
\begin{array}{ll}
\text { (P5) } \max _{\boldsymbol{P}} & \mu \\
\text { s.t. } & R_{m, l}(\boldsymbol{P})-\eta_{m, l}^{i} P_{m, l}(\boldsymbol{P}) \geq \mu, \\
& 0 \leq p_{m, l} \leq p_{\max }, \\
& \frac{p_{m, l}\left|h_{m, l}\right|^{2}}{\sigma_{m}^{2}} \geq \tau_{S F}, \forall m, l .
\end{array}
$$

where the difficulty only lies in the nonconvex constraint (22b).

The left side of constraint (22b) can be further denoted as $f_{i}(\boldsymbol{P})-z_{i}(\boldsymbol{P})$, where $f_{i}(\boldsymbol{P})$ and $z_{i}(\boldsymbol{P})$ are defined as

$f_{i}(\boldsymbol{P})=B_{m} \log _{2}\left(\sum_{k=1}^{S_{m}} p_{m, k}\left|h_{m, k}\right|^{2}+\sigma_{m}^{2}\right)-\eta_{m, l}^{i} P_{m, l}(\boldsymbol{P})$,

$z_{i}(\boldsymbol{P})=B_{m} \log _{2}\left(\sum_{k=1, k \neq l}^{S_{m}} p_{m, k}\left|h_{m, k}\right|^{2}+\sigma_{m}^{2}\right)$.

Constraint (22b) is equivalent to that

$$
f_{i}(\boldsymbol{P})-z_{i}(\boldsymbol{P}) \geq \mu .
$$

Moreover, we can find that $f_{i}(\boldsymbol{P})$ and $z_{i}(\boldsymbol{P})$ are both concave functions with respect to $\boldsymbol{P}$, thus inequality (24) is a DC programming function [35]. Due to the concave feature
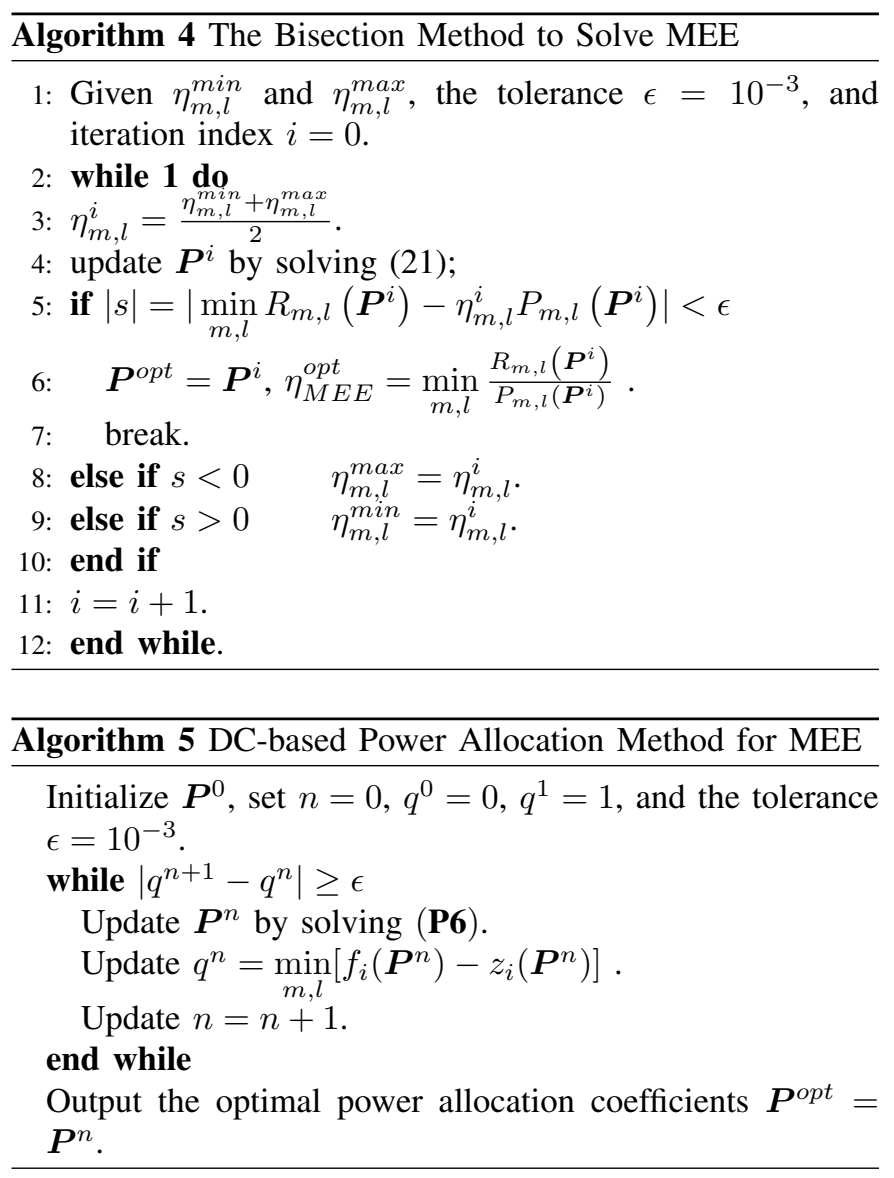

of $z_{i}(\boldsymbol{P})$, we can further approximate it by its upper bound with the first-Taylor expansion as follows:

$$
z_{i}(\boldsymbol{P}) \leq z_{i}\left(\boldsymbol{P}^{n}\right)+\nabla z_{i}^{T}\left(\boldsymbol{P}^{n}\right)\left(\boldsymbol{P}-\boldsymbol{P}^{n}\right),
$$

where $\boldsymbol{P}^{n}$ is the value of $\boldsymbol{P}$ at the $n$-th iteration, $\nabla z_{i}^{T}(\boldsymbol{P})$ represents the gradient of $z_{i}(\boldsymbol{P})$, which can be denoted as

$$
\nabla z_{i}(\boldsymbol{P})=\frac{\nu_{m, k}}{\sum_{k=1, k \neq l}^{S_{m}} p_{m, k}\left|h_{m, k}\right|^{2}+\sigma_{m}^{2}} .
$$

Specifically, $\nu_{m, k}$ is a $S_{m}$-dimensional vector that can be given as

$$
\nu_{m, k}=\left\{\begin{array}{ccc}
0 & \text { if } \quad k=l, \\
\frac{B_{m}\left|h_{m, k}\right|^{2}}{\ln 2} & \text { if } \quad k \neq l .
\end{array}\right.
$$

As a result, the original problem has been converted into the convex form, and during the $n$-th iteration, we need to tackle the following convex problem

(P6)

$$
\begin{aligned}
& \max _{\boldsymbol{P}} \mu \\
& \text { s.t. } f_{i}(\boldsymbol{P})-\left(z_{i}\left(\boldsymbol{P}^{n}\right)+\nabla z_{i}^{T}\left(\boldsymbol{P}^{n}\right)\left(\boldsymbol{P}-\boldsymbol{P}^{n}\right)\right) \geq \mu, \\
& 0 \leq p_{m, l} \leq p_{\max }, \\
& \frac{p_{m, l}\left|h_{m, l}\right|^{2}}{\sigma_{m}^{2}} \geq \tau_{S F}, \forall m, l .
\end{aligned}
$$


Therefore, the detailed process of the DC programming approach to solve power allocations for MEE is outlined as Algorithm 5.

To prove the convergence of the proposed Algorithm 5, we have the following proposition.

Proposition 2. The proposed Algorithm 5 continuously converges to a stationary point of $(\boldsymbol{P 4})$ with given $\eta_{m, l}^{i}$.

Proof: The proof is provided in Appendix G.

\section{Complexity Analysis}

For the energy efficient power allocation for SEE, denote $L_{\max }^{(1)}$ as the maximum iteration number of Algorithm 3, whereas the computational time to solve (P3) by interior point method is proportional to $O\left(r^{3.5} \delta\right)$ [36], where $r$ denotes the number of variables, and $\delta$ is the number of bits needed to represent the entries in the optimization problem. Therefore, the whole complexity to solve SEE is $O\left(L_{\max }^{(1)}(N+1)^{3.5} \delta^{(1)}\right)$.

Concerning MEE, the computational complexity comes from Algorithm 4 and Algorithm 5. Note that by appropriately setting the initial values as $\eta_{m, l}^{\min } \leq \eta_{M E E}^{o p t} \leq \eta_{m, l}^{\max }$, with a given accuracy $\epsilon$, the complexity of the bisection method to solve Algorithm 4 is $\log _{2}\left(\epsilon^{-1}\left(\eta_{m, l}^{\max }-\eta_{m, l}^{\min }\right)\right)$. For Algorithm 5, denote the maximum iteration number as $L_{\max }^{(2)}$, while the complexity of the interior point method to solve (P6) is proportional to $O\left(N^{3.5} \delta^{(2)}\right)$. In conclusion, the computational complexity of MEE is $O\left(\log _{2}\left(\epsilon^{-1}\left(\eta_{m, l}^{\max }-\eta_{m, l}^{\min }\right)\right) L_{\max }^{(2)}\left(N^{3.5} \delta^{(2)}\right)\right)$.

\section{Numerical Results}

In this section, numerical results are provided to evaluate the performance of the proposed algorithms. In the simulations, the simulation parameters are set following LoRa specifications [4]. It is assumed that the LoRa gateway located in the cell center and all the LoRa users are uniformly distributed in a circular range with the radius of $12 \mathrm{~km}$, which is consistent with LoRa characteristics to enable long-range transmission. The number of channels is set to be $M=3$ working at $868 \mathrm{MHz}$. The bandwidth of each channel is set to be $B_{m}=125 \mathrm{kHz}$. We set the path loss factor to be $\alpha=3.5$. Moreover, the duty cycle is set as $1 \%$ by following the LoRaWAN specification. The noise is defined as $\sigma^{2}=-174+10 \log _{10} B_{m} \mathrm{dBm}$. Without loss of generality, we assume that $P_{c}^{1}=\cdots=P_{c}^{N}=P_{c}$, which indicates the same circuit power consumption is adopted for all LoRa users. Besides, the cross correlation factor $\psi$ is a random variable between 0 and 1 , which keeps the same for different SFs within a given channel realization, and $\psi$ varies for different channel realizations.

Fig. 2 illustrates the effectiveness of the proposed user scheduling scheme versus the number of LoRa users with $p_{\max }=20 \mathrm{dBm}$ for both SEE and MEE. The results of the exhaustive search approach and random matching method are provided for comparison. In the "random matching" scheme, the LoRa user randomly chooses a channel among $\boldsymbol{M}$, whereas adopting the proposed SF assignment and power allocation schemes. We observe that the system EE increases monotonically with the number of LoRa users for all the presented methods in the figure, while the max-min EE shows the inverse trend. It is noted that the performance of the proposed low-complexity user scheduling algorithm is very close to that of the exhaustive search method for both cases. Furthermore, the proposed matching algorithm yields much better performance than the random matching scheme. In addition, the gap between the proposed matching algorithm and random matching increases with the larger number of active LoRa users. The reason is that, when the number of active LoRa users increases, the intra-channel interference caused by LoRa users with different SFs can be well controlled by the proposed user scheduling scheme, whereas it cannot be suppressed by random user scheduling. Besides, random user scheduling scheme will schedule channels with poor conditions, which decreases the EE in terms of both SEE and MEE. Furthermore, the proposed matching algorithm plays a more important role in MEE design due to the fact that the max-min EE can be more easily affected by the interference.

In Fig. 3, the performance of the energy efficient power allocation schemes for SEE are evaluated with the active LoRa users ranges from 6 to 16 for $p_{\max }=20 \mathrm{dBm}$. The proposed resource allocation algorithm for SEE is denoted as "Matching+CC", for simplification. To provide a fair comparison, the proposed user scheduling and SF assignment algorithms are adopted for all the three methods presented in the figure. As can be observed from Fig. 3, for SEE, the system EE performs increasing trends with the number of active LoRa users for all three schemes, and especially, the proposed 'matching+CC' algorithm produces the best performance among all three schemes. For instance, when the number of LoRa users is 12, the available system EE for matching $+\mathrm{CC}$ is $8.1 \times 10^{5}$ bits/Joule, while for matching+fixed power and matching+random power are $4.9 \times 10^{5} \mathrm{bits} / \mathrm{Joule}$ and $3.1 \times 10^{5}$ bits/Joule, respectively. Moreover, the advantage of the proposed scheme is more obvious when there are more LoRa users since the gap becomes larger. The reason is that, the intra-channel interference for LoRa users with different SFs cannot be effectively suppressed for random and fixed power allocation.

In Fig. 4, we provide the performance of the proposed power allocation scheme named as "Matching+DC", with the different number of LoRa users for MEE. It shows that the trend for three curves is similar, and the proposed "Matching+DC" scheme yields the best system performance among all three schemes. It is noted that the max-min EE descends with the increasing number of available users, due to the fact that increasing the number of active LoRa users results in more users scheduled in one channel, which increases interference caused by LoRa users allocated in one channel. As a result, the max-min EE declines. In addition, the benefit of the proposed power allocation scheme becomes more obvious with the increasing number of active LoRa users as the gap becomes larger, which is the same as that of Fig. 3.

The system EE performance comparison between SEE and MEE is illustrated in Fig. 5, based on the same random channel realization. We plot the relationship between system $\mathrm{EE}$ and the maximum transmit power for both SEE and MEE. 


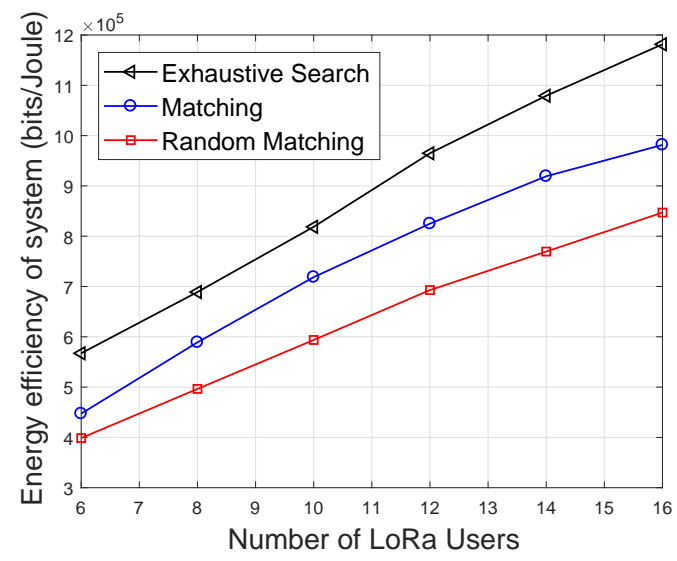

(a) SEE

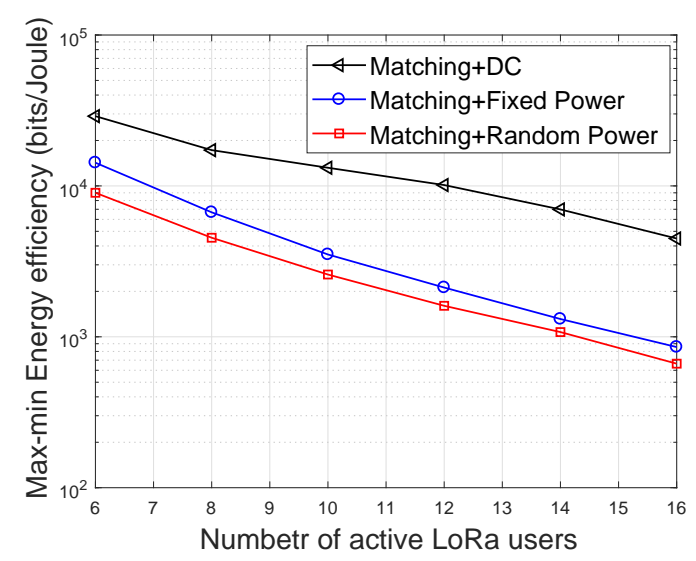

(b) MEE

Fig. 2. Energy efficiency versus number of active LoRa users, N.

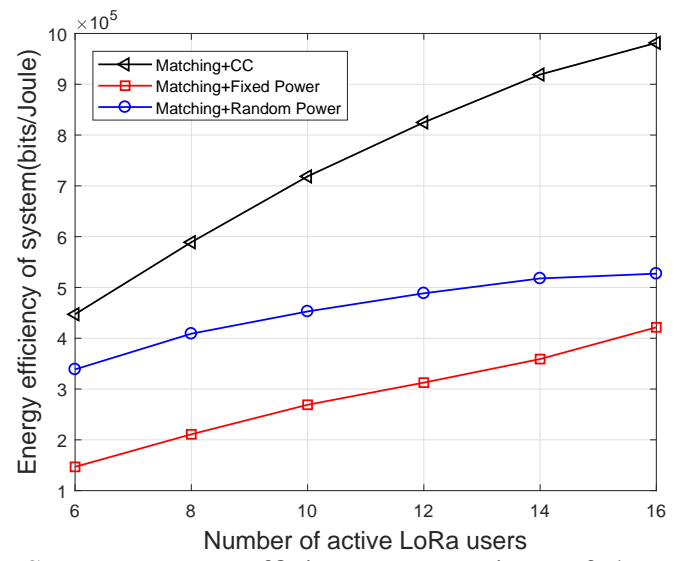

Fig. 3. System energy efficiency comparison of the proposed power allocation schemes versus number of active LoRa users, $\mathrm{N}$.

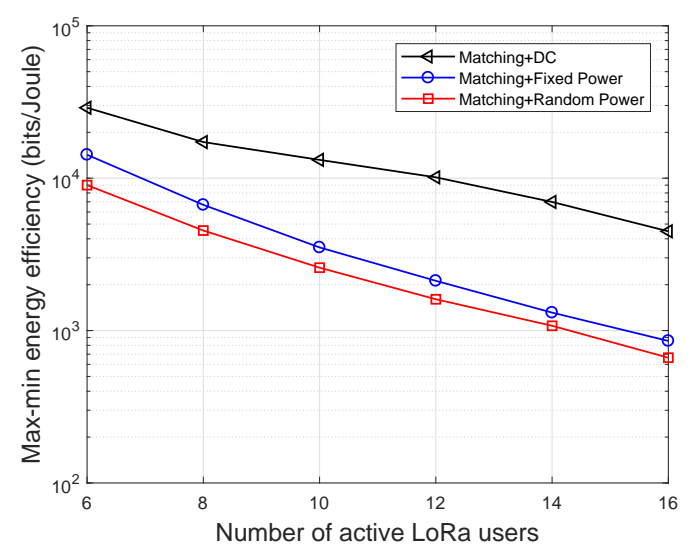

Fig. 4. Max-min energy efficiency comparison of the proposed power allocation schemes versus number of active LoRa users, N.

We can see that the SEE design achieves significantly higher system EE compared with MEE design. The system EE gap between $P_{c}=0.01 \mathrm{~W}$ and $P_{c}=0.05 \mathrm{~W}$ decreases with $p_{\max }$ for both SEE and MEE design. This is because the proportion of circuit power consumption decreases with the increasing $p_{\max }$, and the effect of circuit power consumption is more obvious in the low power regime. Moreover, when $P_{c}=0.01$ $\mathrm{W}$, the system EE firstly increases with $p_{\max }$ and reaches the peak at $22 \mathrm{dBm}$, and then it decreases.

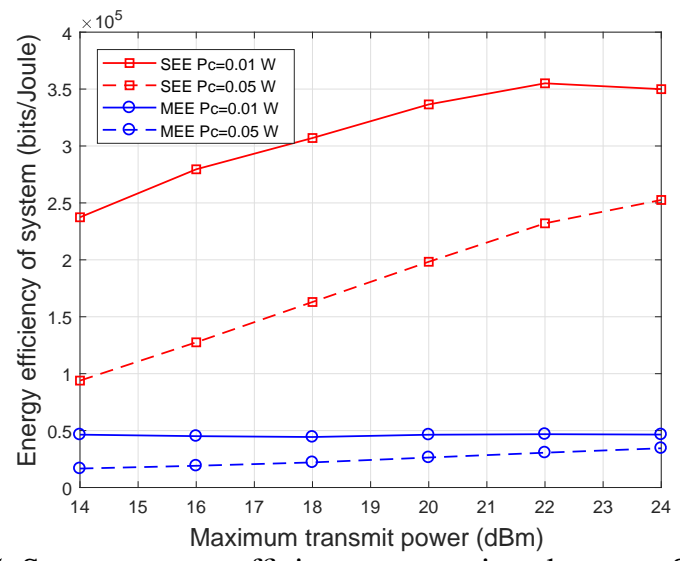

Fig. 5. System energy efficiency comparison between SEE and MEE versus $p_{\max }$.

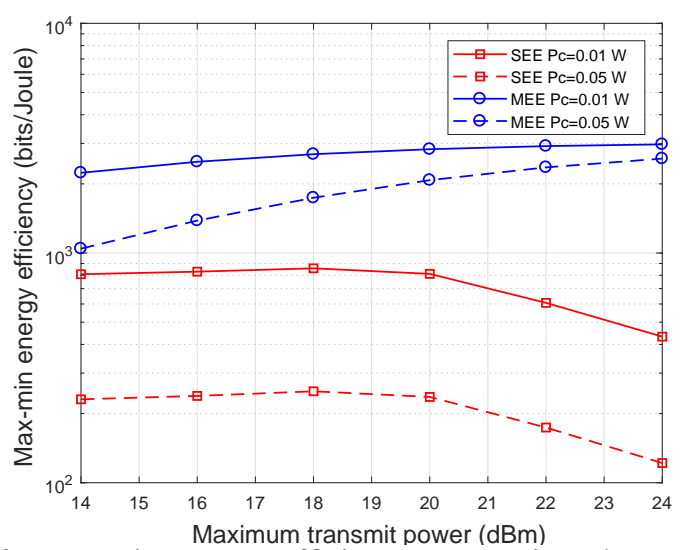

Fig. 6. Max-min energy efficiency comparison between SEE and MEE versus $p_{\max }$.

Fig. 6 is plotted to compare the max-min EE performance for SEE and MEE with the given random channel, which indicates that the max-min EE for MEE slightly improves with the increasing $p_{\max }$. However, for SEE, the max-min EE firstly keeps constant, and then decreases. The reason is that, when $p_{\max }$ becomes larger, more resources will be allocated to users with bad channel conditions, which guarantees fairness among users for MEE design. On the other hand, to improve system performance, SEE will schedule more power to users in good channel conditions and sacrifice the benefit of bad users, 
and the corresponding max-min EE decreases. Moreover, for MEE, the gap between $P_{c}=0.01 \mathrm{~W}$ and $P_{c}=0.05 \mathrm{~W}$ becomes smaller with $p_{\max }$, due to the reason that $p_{\max }$ dominates $P_{c}$ in the high power regime. Combining Fig. 5 and Fig. 6, it can be seen that SEE and MEE have completely different preferences in EE design, and the network can adopt the corresponding strategies according to the system requirement.

\section{CONCLUSION}

In this paper, we have investigated the uplink LoRa networks to maximize the $\mathrm{EE}$ of the whole network and the minimal EE of LoRa users, named as SEE and MEE, respectively. Particularly, we decompose the formulated problems into three sub-problems, including user scheduling, SF assignment, and power allocation. Moreover, we have proposed a low-complexity user scheduling scheme to solve the channel assignment problem by formulating it as a many-toone two-sided matching problem with peer effects. The SF is assigned to LoRa users scheduled on the same channel based on the distance between LoRa user and gateway, which is obtained from the stable matching. Moreover, for energy efficient power allocation to maximize SEE, we approximate the fractional nonconvex function by its lower bound, which can be further transformed into convex approximations with Charnes-Cooper transformation. While to maximize MEE, an iterative method based on generalized fractional programming and DC programming has been proposed. Numerical results have shown that the proposed matching algorithms and power allocation schemes outperform the existing schemes in terms of both SEE and MEE.

\section{APPENDIX A PROOF OF THEOREM 1}

Take SEE as an example, Theorem 1 can be proved by considering two cases, i.e, $\Lambda_{\max }=1$ and $\Lambda_{\max }>1$.

- When $\Lambda_{\max }=1$, the original SEE reduces to the joint channel and power allocations for system EE maximization problem in an OFDMA system, the NP-harness has already been proved in [37].

- For the case of $\Lambda_{\max }>1$, we prove that SEE is NPhard even without considering power allocations. We construct a case of SEE with given power allocation coefficients and the NP-hardness can be proved by establishing the equivalence between the constructed instance and 3-dimensional matching problem, which is known to be NP-hard. The instance with $N$ LoRa users, $M$ channels, and $\Lambda_{\max }=2$ is considered. Let $\mathfrak{X}$ and $\mathfrak{Y}$ be two different sets with $|\mathfrak{X}|=|\mathfrak{Y}|=\frac{N}{2}$ and $V$ be a subset of $M \times \mathfrak{X} \times \mathfrak{Y}$. Assuming that any tripe $V_{i}=\left(m_{i}, x_{i}, y_{i}\right) \in V$, which means LoRa users $x_{i} \in \mathfrak{X}$, $y_{i} \in \mathfrak{Y}$ are selected on channel $m_{i} \in M$. With given power allocation coefficients, denote the maximized sum rate with any given $V_{i}$ as $\mathfrak{R}_{V_{i}}$. Hence, we just need to verify the 3-dimensional problem if there exists $V^{\prime} \subset V$, satisfying that 1) $m_{1} \neq m_{2}, x_{1} \neq x_{2}$, and $y_{1} \neq y_{2}$ for any two triples $\left(m_{1}, x_{1}, y_{1}\right) \in V^{\prime}$ and $\left(m_{2}, x_{2}, y_{2}\right) \in V^{\prime}$. 2) $V^{\prime}=\min \left\{M, \frac{N}{2}\right\}$.

According to the definition, if the feasibility problem is NPhard, the original problem is also NP-hard [38]. Therefore, denote the sum rate for any triple as $R_{V_{i}^{\prime}}$, given the power allocations, the sum rate feasibility problem can be expressed as $\sum_{i=1}^{i=V^{\prime}} R_{V_{i}^{\prime}} \leq \epsilon$, where $\epsilon$ is a given constant. When $\epsilon$ becomes positive infinity, an instance of the feasibility problem corresponds to a 3-dimensional matching problem, then a special case of the original SEE is NP-harness, which proves the original SEE is NP-hard. The proof for MEE is similar and omitted here. The proof is completed.

\section{APPENDIX B \\ PROOF OF THEOREM 2}

Theorem 2 is proved from two aspects. Firstly, the number of possible swap matching operations is finite since only a limited number of LoRa users can occupy the same channel. In addition, Due to the feature of swap matching given by Definition 2, if a swap-matching is approved, the achievable data rates of any player, i.e., $U_{l}$ and $S C_{m}$, will not decrease by employing a swap matching, and the data rates of at least one player will increase. Therefore, the corresponding objectives, i.e., (7a) and (8a), will increase after each swap matching operation. The spectrum resources are limited, which restricts the upper bound of energy efficiency. Hence, there is a swap matching after which no further energy efficiency is improved and Algorithm 1 converges to a $2 E S$ matching. The proof is completed.

\section{APPENDIX C \\ PROOF OF THEOREM 3}

The initialization step is a deferred acceptance algorithm, the complexity depends on the process of user proposing, which is up to $O(M N)$ in the worst case. Besides, the computational complexity of the swap matching step lies in the number of iterations and swap operations. In each iteration, for any channel $S C_{m}$, the maximum assigned users is $\Lambda_{\max }$. For user $U_{j}$, there exists $(M-1)$ possible swap-blocking pairs in $\varphi_{l}^{j}$. The potential combinations for $\varphi_{l}^{j}$ with $j$ fixed is $\Lambda_{\max }(M-1)$. Since there are $N$ LoRa users, we can conclude that the number of swap matchings is $\Lambda_{\max } N(M-1)$ during each iteration. Considering the number of iterations, the total complexity of swap matching is $O\left(\frac{1}{2} I \Lambda_{\max } N(M-1)\right)$. Combining the above two phases, the complexity of Algorithm 1 is $O\left(M N+\frac{1}{2} I \Lambda_{\max } N(M-1)\right)$ in the worst case. The proof is completed.

\section{APPENDIX D PROOF OF PROPOSITION 1}

With inequality (11) and equation (14), the original SEE has been transformed into a concave-convex problem, which implies that KKT conditions are sufficient and any local maximum is the global maximum [39]. Besides, a feasible point set $\left\{\tilde{x}_{m, l}^{t}\right\}$ and $\left\{\phi^{t}\right\}$ at iteration $t$ can be obtained by solving (P3), the power allocated to each user is calculated 
as $p_{m, l}{ }^{t}=e^{\frac{\tilde{x}_{m, l}^{t}}{\phi^{t}}}$. The system EE $\eta^{t}$ can be further obtained according to (7a). Moreover, $(\mathbf{P 3})$ is concave in $(\tilde{x}, \phi)$, with the interior point method, we can derive that $\eta^{t} \leq \eta^{t+1}$ which means the Algorithm 3 converges. The proof is completed.

\section{APPENDIX E \\ PROOF OF THEOREM 4}

The proof can be proved from two aspects. We first prove the necessity of Theorem 4. From equation (19), we can deduce that

$$
\eta_{M E E}^{o p t}=\min _{m, l} \frac{R_{m, l}\left(\boldsymbol{P}^{o p t}\right)}{P_{m, l}\left(\boldsymbol{P}^{o p t}\right)} \geq \min _{m, l} \frac{R_{m, l}(\boldsymbol{P})}{P_{m, l}(\boldsymbol{P})},
$$

which is equivalent to the following

$$
\begin{array}{r}
\min _{m, l}\left[R_{m, l}(\boldsymbol{P})-\eta_{M E E}^{o p t} P_{m, l}(\boldsymbol{P})\right] \leq 0, \\
\min _{m, l}\left[R_{m, l}\left(\boldsymbol{P}^{o p t}\right)-\eta_{M E E}^{o p t} P_{m, l}\left(\boldsymbol{P}^{o p t}\right)\right]=0 .
\end{array}
$$

Therefore, the maximum value for the left side of (E.2a) can be achieved if and only if $\boldsymbol{P}=\boldsymbol{P}^{\text {opt }}$, which completes the necessity proof.

The we prove the sufficiency of Theorem 4. Assuming $\boldsymbol{P}^{\prime}$ as the optimal power allocation to (19), then for any feasible power allocation coefficient $\boldsymbol{P}$, we have the following function

$$
\begin{aligned}
\min _{m, l} & {\left[R_{m, l}(\boldsymbol{P})-\eta_{M E E}^{o p t} P_{m, l}(\boldsymbol{P})\right] \leq } \\
& \min _{m, l}\left[R_{m, l}\left(\boldsymbol{P}^{\prime}\right)-\eta_{M E E}^{o p t} P_{m, l}\left(\boldsymbol{P}^{\prime}\right)\right]=0 .
\end{aligned}
$$

As a result, we can obtain that

$$
\eta_{M E E}^{o p t}=\min _{m, l} \frac{R_{m, l}\left(\boldsymbol{P}^{\prime}\right)}{P_{m, l}\left(\boldsymbol{P}^{\prime}\right)},
$$

which means $\boldsymbol{P}^{\prime}$ is the optimal power allocation of the original problem. The proof is completed.

\section{APPENDIX F \\ PROOF OF THEOREM 5}

Let us assume that $\boldsymbol{P}^{1}$ and $\boldsymbol{P}^{2}$ be the optimal power solution corresponding to given max-min EE $\eta_{m, l}^{1}$ and $\eta_{m, l}^{2}$, with the condition $\eta_{m, l}^{1}>\eta_{m, l}^{2}$, then we can deduce the following

$$
\begin{aligned}
\pi\left(\eta_{m, l}^{1}\right) & =\max _{\boldsymbol{P}} \min _{m, l}\left[R_{m, l}(\boldsymbol{P})-\eta_{m, l}^{1} P_{m, l}(\boldsymbol{P})\right] \\
& =\min _{m, l}\left[R_{m, l}\left(\boldsymbol{P}^{1}\right)-\eta_{m, l}^{1} P_{m, l}\left(\boldsymbol{P}^{1}\right)\right] \\
& <\min _{m, l}\left[R_{m, l}\left(\boldsymbol{P}^{1}\right)-\eta_{m, l}^{2} P_{m, l}\left(\boldsymbol{P}^{1}\right)\right] \\
& \leq \min _{m, l}\left[R_{m, l}\left(\boldsymbol{P}^{2}\right)-\eta_{m, l}^{2} P_{m, l}\left(\boldsymbol{P}^{2}\right)\right]=\pi\left(\eta_{m, l}^{2}\right)
\end{aligned}
$$

where inequality (F.1c) is derived from the condition that $\eta_{m, l}^{1}>\eta_{m, l}^{2}$, (F.1d) is obtained due to the fact $\boldsymbol{P}^{2}$ is the optimal solution to $\eta_{m, l}^{2}$. Hence, $\pi\left(\eta_{m, l}\right)$ is monotonically decreasing with $\eta_{m, l}$. In addition, incorporating the conclusion gained from Theorem 4, we can easily derive the property $i i$ ) of Theorem 5. The proof is completed.

\section{APPENDIX G \\ PROOF OF PROPOSITION 2}

To prove the convergence of Algorithm 5, we need to confirm that the optimal solution to problem (P6) at $n$-th iteration is also a feasible point of the iteration $n+1$. From the characteristic of inequality (28b), denote $\boldsymbol{P}^{*}$ as the optimal solution to $(\mathbf{P 6})$ at iteration $n$, the following result can be obtained:

$$
\begin{aligned}
u & \leq q^{n} \\
& =\min _{m, l} f_{i}\left(\boldsymbol{P}^{*}\right)-\left(z_{i}\left(\boldsymbol{P}^{n}\right)+\nabla z_{i}^{T}\left(\boldsymbol{P}^{n}\right)\left(\boldsymbol{P}^{*}-\boldsymbol{P}^{n}\right)\right) \\
& =\min _{m, l} f_{i}\left(\boldsymbol{P}^{n+1}\right)-\left(z_{i}\left(\boldsymbol{P}^{n}\right)+\nabla z_{i}^{T}\left(\boldsymbol{P}^{n}\right)\left(\boldsymbol{P}^{n+1}-\boldsymbol{P}^{n}\right)\right) \\
& \leq \min _{m, l}\left[f_{i}\left(\boldsymbol{P}^{n+1}\right)-z_{i}\left(\boldsymbol{P}^{n+1}\right)\right]=q^{n+1}, \quad \text { (G.1c) }
\end{aligned}
$$

where the equality (G.1c) holds as $\boldsymbol{P}^{n+1}$ is the optimal solution of $n$-th iteration. Besides, inequality (G.1d) can be derived with inequality (25), since the first-Taylor approximation is an upper bound of $z_{i}(\boldsymbol{P})$.

In conclusion, we can obtain that the objective value at the iteration $n+1$ is larger or equal to that achieved from the $n$-th iteration, which proves the convergence of Algorithm 5.

Moreover, denote constraint (28b) as $\varsigma(\boldsymbol{P})=f_{i}(\boldsymbol{P})-$ $\left(z_{i}\left(\boldsymbol{P}^{n}\right)+\nabla z_{i}^{T}\left(\boldsymbol{P}^{n}\right)\left(\boldsymbol{P}-\boldsymbol{P}^{n}\right)\right)$. Since Algorithm 5 converges, then $\boldsymbol{P}^{n}=\boldsymbol{P}^{n+1}$ when $n \rightarrow \infty$. The first-order optimality condition [40] can be written as

$$
\begin{array}{rlr}
\nabla \varsigma^{T}\left(\boldsymbol{P}^{n}\right)\left(\boldsymbol{P}-\boldsymbol{P}^{n}\right) & =\left(\nabla f_{i}^{T}\left(\boldsymbol{P}^{n}\right)-\nabla z_{i}^{T}\left(\boldsymbol{P}^{n}\right)\right)\left(\boldsymbol{P}-\boldsymbol{P}^{n}\right) \\
& =\nabla \varsigma^{T}\left(\boldsymbol{P}^{n+1}\right)\left(\boldsymbol{P}-\boldsymbol{P}^{n+1}\right) & (\mathrm{G} .2 \mathrm{~b}) \\
& \leq 0 . & (\mathrm{G} .2 \mathrm{c})
\end{array}
$$

where equality (G.2a) holds since $\varsigma(\boldsymbol{P})$ has the same gradient value as $f_{i}(\boldsymbol{P})-z_{i}(\boldsymbol{P})$ at the point $\boldsymbol{P}^{n}$. (G.2b) is derived by replacing $\boldsymbol{P}^{n}$ with $\boldsymbol{P}^{n+1}$, and (G.2c) is gained for $\boldsymbol{P}=\boldsymbol{P}^{n}$.

Consequently, the first-order optimality condition of problem (P4) is confirmed. According to [41, Proposition 3], we can conclude that the result obtained from Algorithm 5 satisfies KKT conditions and is a stationary point of (P4). The proof is completed.

\section{REFERENCES}

[1] B. Su, Z. Qin, and Q. Ni, "Energy efficient resource allocation for uplink LoRa networks," in Proc. IEEE GLOBECOM, Abu Dhabi, UAE, Dec. 2018, pp. 1-7.

[2] Q. M. Qadir, T. A. Rashid, N. K. Al-Salihi, B. Ismael, A. A. Kist, and Z. Zhang, "Low power wide area networks: A survey of enabling technologies, applications and interoperability needs," IEEE Access, vol. 6, pp. 77 454-77473, Nov. 2018.

[3] Z. Qin, F. Y. Li, G. Y. Li, J. A. McCann, and Q. Ni, "Low-power widearea networks for sustainable IoT," IEEE Wireless Commun., vol. 26, no. 3, pp. 140-145, June 2019.

[4] N. Sornin, M. Luis, T. Eirich, T. Kramp, and O. Hersent, "LoRaWAN specification, 1-82,” Jan. 2015. [Online]. Available: https://www.loraalliance.org/portals/0/specs/LoRaWAN\%20Specification\% 201R0.pdf.

[5] U. Raza, P. Kulkarni, and M. Sooriyabandara, "Low power wide area networks: An overview," IEEE Commun. Surveys Tuts., vol. 19, no. 2, pp. 855-873, Jan. 2017. 
[6] O. Georgiou and U. Raza, "Low power wide area network analysis: Can LoRa scale?" IEEE Wireless Commun. Lett., vol. 6, no. 2, pp. 162-165, Jan. 2017.

[7] A. Waret, M. Kaneko, A. Guitton, and N. E. Rachkidy, "LoRa throughput analysis with imperfect spreading factor orthogonality," IEEE Wireless Commun. Lett., vol. 8, no. 2, pp. 408-411, Oct. 2018.

[8] D. Croce, M. Gucciardo, S. Mangione, G. Santaromita, and I. Tinnirello, "Impact of LoRa imperfect orthogonality: Analysis of link-level performance," IEEE Commun. Lett., vol. 22, no. 4, pp. 796-799, Apr. 2018.

[9] L. Amichi, M. Kaneko, E. H. Fukuda, N. E. Rachkidy, and A. Guitton, "Joint allocation strategies of power and spreading factors with imperfect orthogonality in LoRa networks," IEEE Trans. Commun., Early Access, 2020.

[10] V. Hauser and T. Hegr, "Proposal of adaptive data rate algorithm for LoRaWAN-based infrastructure," in IEEE 5th Intl. Conf. FiCloud, Aug. 2017, pp. 85-90.

[11] S. Li, U. Raza, and A. Khan, "How agile is the adaptive data rate mechanism of LoRaWAN?" in Proc. IEEE GLOBECOM, Abu Dhabi, UAE, Dec. 2018, pp. 206-212.

[12] K. Q. Abdelfadeel, V. Cionca, and D. Pesch, "Fair adaptive data rate allocation and power control in LoRaWAN," pp. 14-15, June 2018.

[13] K. Mikhaylov, J. Petaejaejaervi, and T. Haenninen, "Analysis of capacity and scalability of the LoRa low power wide area network technology," in Proc. 22nd Eur. Wireless Conf. , Oulu, Finland, May 2016, pp. 1-6.

[14] Z. Qin and J. A. McCann, "Resource efficiency in low-power wide-area networks for IoT applications," in Proc. IEEE GLOBECOM, Singapore, Dec. 2017, pp. 1-7.

[15] J. Haxhibeqiri, F. Van den Abeele, I. Moerman, and J. Hoebeke, "LoRa scalability: A simulation model based on interference measurements,' Sensors, vol. 17, no. 6, p. 1193, May 2017.

[16] Y. Hao, Q. Ni, H. Li, and S. Hou, "On the energy and spectral efficiency tradeoff in massive MIMO-enabled hetnets with capacity-constrained backhaul links," IEEE Trans. Commun., vol. 65, no. 11, pp. 4720-4733, Nov. 2017.

[17] M. Bashar, K. Cumanan, A. G. Burr, H. Q. Ngo, E. G. Larsson, and P. Xiao, "On the energy efficiency of limited-backhaul cell-free Massive MIMO," in IEEE Intl. Conf. Commun. (ICC), May 2019, pp. 1-7.

[18] S. Li, Q. Ni, Y. Sun, G. Min, and S. Al-Rubaye, "Energy-efficient resource allocation for industrial cyber-physical IoT systems in $5 \mathrm{G}$ era," IEEE Trans. Ind. Informat., vol. 14, no. 6, pp. 2618-2628, June 2018.

[19] C. C. Zarakovitis, Q. Ni, and J. Spiliotis, "Energy-efficient green wireless communication systems with imperfect CSI and data outage," IEEE J. Sel. Areas Commun., vol. 34, no. 12, pp. 3108-3126, Dec. 2016.

[20] H. Al-Obiedollah, K. Cumanan, J. Thiyagalingam, A. G. Burr, Z. Ding, and O. A. Dobre, "Energy efficiency fairness beamforming designs for MISO NOMA systems," in IEEE WCNC19, Marakkech, Morocco, Apr. 2019, pp. 1-7.

[21] Q. Wu, M. Tao, D. W. K. Ng, W. Chen, and R. Schober, "Energyefficient resource allocation for wireless powered communication networks," IEEE Trans. Wireless Commun., vol. 15, no. 3, pp. 2312-2327, Mar. 2016.

[22] H. Al-Obiedollah, K. Cumanan, J. Thiyagalingam, A. G. Burr, Z. Ding, and O. A. Dobre, "Energy efficient beamforming design for MISO non-orthogonal multiple access systems," IEEE Trans. Commun. Early Access, vol. 67, no. 6, pp. 4117-4131, June 2019.

[23] L. Venturino, A. Zappone, C. Risi, and S. Buzzi, "Energy-efficient scheduling and power allocation in downlink OFDMA networks with base station coordination," IEEE Trans. Wireless Commun., vol. 14, no. 1, pp. 1-14, Jan. 2015

[24] Y. Li, M. Sheng, C. W. Tan, Y. Zhang, Y. Sun, X. Wang, Y. Shi, and J. Li, "Energy-efficient subcarrier assignment and power allocation in OFDMA systems with max-min fairness guarantees," IEEE Trans. Commun., vol. 63, no. 9, pp. 3183-3195, Sept. 2015.

[25] I. Chih-Lin, C. Rowell, S. Han, Z. Xu, G. Li, and Z. Pan, "Toward green and soft: a $5 \mathrm{G}$ perspective," IEEE Commun. Mag., vol. 52, no. 2, pp. 66-73, Feb. 2014

[26] S. Rani, R. Talwar, J. Malhotra, S. H. Ahmed, M. Sarkar, and H. Song, "A novel scheme for an energy efficient Internet of Things based on wireless sensor networks," Sensors, vol. 15, no. 11, pp. 28603-28626, Nov. 2015.

[27] C. Yang, J. Li, Q. Ni, A. Anpalagan, and M. Guizani, "Interferenceaware energy efficiency maximization in 5G ultra-dense networks," IEEE Trans. Commun., vol. 65, no. 2, pp. 728-739, Jan. 2017.

[28] M. C. Bor, U. Roedig, T. Voigt, and J. M. Alonso, "Do LoRa lowpower wide-area networks scale?" in Proc. ACM Intl. Conf. Modeling, Analysis Simul. Wireless Mobile Systems, Nov. 2016, pp. 59-67.
[29] M. Dianati, X. Shen, and K. Naik, "Cooperative fair scheduling for the downlink of CDMA cellular networks," IEEE Trans. Veh. Technol., vol. 56, no. 4, pp. 1749-1760, July 2007.

[30] E. Bodine-Baron, C. Lee, A. Chong, B. Hassibi, and A. Wierman, "Peer effects and stability in matching markets," in Proc. 4th Symp. Algorithmic Game Theory (SAGT), Oct. 2011, pp. 117-129.

[31] D. Gale and L. S. Shapley, "College admissions and the stability of marriage," The American Mathematical Monthly, vol. 69, no. 1, pp. 9-15, 1962

[32] J. Papandriopoulos and J. S. Evans, "Low-complexity distributed algorithms for spectrum balancing in multi-user DSL networks," in Proc. IEEE Intl. Commun. Conf. (ICC), Istanbul, Turkey, vol. 7, 2006, pp. 3270-3275.

[33] A. Charnes and W. W. Cooper, "Programming with linear fractional functionals," Naval Research Logistics Quarterly, vol. 10, no. 1, pp. 273-274, 1963.

[34] M. Grant, S. Boyd, and Y. Ye, "CVX: Matlab software for disciplined convex programming," 2008.

[35] N. Vucic, S. Shi, and M. Schubert, "DC programming approach for resource allocation in wireless networks," in Proc. Int. Symp. Modeling Optim. Ad Hoc Wireless Netw. (WiOpt), June 2010, pp. 380-386.

[36] I. J. Lustig, R. E. Marsten, and D. F. Shanno, "Interior point methods for linear programming: Computational state of the art," ORSA J. Computing, vol. 6, no. 1, pp. 1-14, 1994.

[37] Y. Liu and Y. Dai, "On the complexity of joint subcarrier and power allocation for multi-user OFDMA systems,"' IEEE Trans. Signal Process., vol. 62, no. 3, pp. 583-596, Nov. 2014.

[38] M. R. Garey, "A guide to the theory of NP-Completeness," Computers and intractability, 1979.

[39] G. R. Lanckriet and B. K. Sriperumbudur, "On the convergence of the concave-convex procedure," in Proc. Adv. Neural Inf. Process. Syst., 2009, pp. 1759-1767.

[40] S. Boyd and L. Vandenberghe, Convex optimization. Cambridge university press, 2004.

[41] A. Zappone, E. Bjrnson, L. Sanguinetti, and E. Jorswieck, "Globally optimal energy-efficient power control and receiver design in wireless networks," IEEE Trans. Signal Process., vol. 65, no. 11, pp. 2844-2859, June 2017. 